\title{
Aeroelastic Modeling and Simulation of High-Speed Flexible Vehicles
}

\author{
Ryan C. Kitson* and Carlos E. S. Cesnik ${ }^{\dagger}$ \\ University of Michigan, Ann Arbor, MI, 48109, USA
}

\begin{abstract}
Air-to-air intercept flight requires vehicles to maintain superior maneuverability and agility to capture their target at supersonic speeds. Aggressive maneuvers create complex flows around the vehicle that lead to very high loads and interaction between the aerodynamics, structural dynamics and flight dynamics. These interactions become significant for flexible vehicles with larger deformations such as the very slender structures that may be used for air-to-air missions. A representative vehicle model has been developed based on reduced order and fundamental tools to enable the study of the aeroelastic response of a maneuvering vehicle. This paper presents the development of the vehicle model and the computational framework to analyze the aeroelastic response of the maneuvering vehicle in free flight. The vehicle response to control inputs for various values of vehicle bending stiffness and distribution is presented to investigate the variation of performance with vehicle flexibility. The results show that the vehicle loading, maneuverability, and agility are all dependent on the vehicle structural configuration. Therefore, accurate analyses of future designs are dependent on vehicle modeling as flexible structures. Understanding the aeroelastic response of a flexible munition could lead to vehicle stiffness becoming an additional design parameter to improve maneuverability and agility.
\end{abstract}

\section{Nomenclature}

$C \quad$ Damping matrix of the aeroelastic system

$F_{D A C S}$ Force applied by the DACS

I DACS Impulse

$K \quad$ Reduced stiffness matrix of the structural system

$L_{r e f} \quad$ Reference length, the vehicle length

$M \quad$ Mass matrix of the aeroelastic system

$Q \quad$ Generalized forces of the aeroelastic system

a Speed of sound

$f \quad$ DACS frequency

$i \quad$ Free-free vibration mode index $(i=1 \ldots n)$

$n \quad$ Total number of free-free vibration modes

$p \quad$ Pressure

$t \quad$ Time

$t_{d} \quad$ DACS time duration

$u \quad$ Displacement vector

$w \quad$ Surface velocity perpendicular to the flow

$\Delta() \quad$ Change in quantity

$\Phi \quad$ Free-free vibration mode

$\beta \quad$ Rigid body translational velocity vector

$\ddot{\gamma} \quad$ Flight path angle acceleration

$\dot{\gamma} \quad$ Flight path angle velocity

$\eta \quad$ Free-free vibration mode amplitude

*Ph.D. Candidate, Department of Aerospace Engineering, kitson@umich.edu

${ }^{\dagger}$ Professor, Department of Aerospace Engineering, cesnik@umich.edu; AIAA Fellow 


\section{Introduction}

$\mathrm{T}$

HERE is a premium placed on vehicle maneuverability and agility to capture evading targets. Current air-to-air vehicles are designed to maneuver immediately after launch and separation from the aircraft in the high subsonic and low supersonic regimes. ${ }^{1}$ The vehicle then begins to accelerate and close in while performing minor maneuvers to track the target. The vehicle is traveling at high supersonic speeds during the final phase of the trajectory, which prohibits large final adjustments to its flight path. This is a weakness that can be exploited by the target, which limits the effectiveness of the air-to-air vehicle. Significant changes to the trajectory of a supersonic vehicle require very high angles of attack (AoA) leading to very high pressures on the slender structure. Therefore, design and analysis of next generation intercept vehicles requires a better understanding of the coupled interactions between the flexible structure, aerodynamics, control system and flight dynamics.

Air vehicle designs attempt to minimize common parameters such as weight and drag while maximizing performance. One approach to minimizing the weight is to use less structural material or a different lightweight material for the vehicle structure. Modifying the structural configuration to reduce weight may lead to larger structural deformations and stronger coupling between the aerodynamics and structure. There is a significant amount of research on maneuvering projectiles at supersonic speeds that stems from the Army research community, but this work focuses on rigid projectiles or structures with only one discrete degree of freedom. Costello and Argarwalla showed how introducing a hinge near the nose could lead to a passive control mechanism that improved stability, but did not address any other hinge locations or multiple hinges similar to a continuous structure. ${ }^{2}$ Including structural degrees of freedom requires a coupled aeroelastic solution to accurately measure the effects of increasing flexibility. Recent work by Yao, Wu, and Yang looked at maneuvering flexible vehicles and the effects of varying vehicle flexibility that suggested vehicle performance is significantly affected by structural stiffness, but used constant linear coefficients for the aerodynamic forces and only particular mode shape was used for the calculations. ${ }^{3}$ Additional study is needed to understand the effect of flexibility and structural configuration on the aeroelastic response of maneuvering high speed vehicles.

Modification to the vehicle control system can also affect the performance of slender high speed vehicles. A divert and attitude control system (DACS) consisting of reaction jets can improve the steady state drag and control effectiveness of the vehicle over conventional fin surfaces. Jets in a supersonic crossflow introduce complex flows around the vehicle that can amplify or reduce the effective force and moment on the vehicle. Roger presented a thorough review of the experimental work focused on jet interaction and showed how the forces applied to the structure can be significantly amplified by the jet interaction with the flow and body. ${ }^{4}$ However, there is a lack of information on jet interaction with a flexible structure. Vehicle deformation could be seen as a change in local angle of attack and is suspected to have an effect on the amplification factors. Therefore it is important to develop a method of modeling flexible vehicles with a jet control system in order to understand the effect on the overall response.

Multiple physical phenomena associated with a supersonic maneuvering vehicle must be modeled to fully understand the flight dynamics, trajectory, and ultimately the performance of the vehicle. Aggressive maneuvers for vehicles are difficult to conduct experimentally, which leads to emphasis placed on numerical analysis. High fidelity multidisciplinary analysis tools, such as Kestrel, are available to analyze maneuvering flexible vehicles. ${ }^{5}$ However, these tools have a high computational cost, complexity and possible robustness issues. Reduced order and fundamental models are an effective way to capture the relevant physical phenomena and couplings of the full problem while significantly reducing computational cost and complexity and increasing computational robustness. Reduced order aerodynamic models have been shown to achieve a high level of accuracy with a low computational cost. ${ }^{6-8}$ In addition, reduced order modeling frameworks that couple the aerodynamic and structural reduced order models for unsteady aeroelastic problems have been used for vehicle design, ${ }^{9,10}$ but these frameworks are not configured to analyze a flexible supersonic vehicle performing high-g maneuvers. A reduced order modeling framework presented by Klock and Cesnik 
couples the reduced order models of various disciplines to simulate the flexible vehicle flight response and is modified for this study to include a jet interaction capability for the DACS. ${ }^{11}$

A flexible supersonic vehicle model is developed and used within a coupled aeroelastic framework to study the effects of adding flexibility, modifying the structural configuration, and using a DACS system to perform high-g maneuvers. The vehicle properties have been chosen to be representative of the air intercept vehicle class such as the Sidewinder and Advanced Medium Range Air to Air Missile (AMRAAM). However, the vehicle is unconventional in some aspects in anticipation of future air-to-air vehicle technology studies. Specifically, the vehicle is an axisymmetric body without any control surfaces or wings resembling a slender cone-cylinder-flare geometry. For control authority, a set of reaction jets are assumed to be located near the nose of the vehicle. Uniform structural properties have been used to demonstrate the effect of deformation on vehicle performance. In addition, representative structural properties were used to demonstrate their effect on the vehicle free-free vibration characteristics and vehicle response. The vehicle response to a prescribed control force was recorded for varying values of vehicle flexibility and varying structural properties. The response of the system and the effects of increased flexibility are highlighted by comparing the structural and flight dynamic response for each stiffness value and structural model. Analyzing the response of the vehicle for various levels of flexibility, different structural configurations, and different control inputs can show trends that might affect a preliminary design phase for a new vehicle.

\section{Methods}

Reduced order and fundamental models are used for the aeroelastic simulation of the slender high speed maneuvering vehicle. The structural dynamics, aerodynamics, and DACS models are included in a previously established partitioned simulation framework presented by Klock and Cesnik. ${ }^{11}$ This simulation framework calculates the aeroelastic equations of motion coupled with the flight dynamics equations of motion and marches the solution forward in time. The reduced order modeling approach facilitates experimentation in many areas of the design space which is used to gain an understanding of the vehicle response.

Reduced order models have been used throughout previous work regarding unsteady aeroelastic problems. Crowell and McNamara showed how reduced order models for unsteady aerodynamics had large decreases in computation time compared to a CFD solver while maintaining relatively high accuracy. ${ }^{8}$ McNamara et al. showed that using local piston theory was able to calculate the flutter Mach number with low error relative to a Navier-Stokes CFD solution for a two-dimensional test case. ${ }^{7}$ Klock and Cesnik showed how a collection of reduced order models for the aerodynamics, thermodynamics and structural dynamics could be coupled to the flight dynamics equations for a vehicle in free flight. ${ }^{11}$ This partitioned approach is the basis for the present work and applied to the high speed slender vehicle. The computational cost is reduced by eliminating the large number of degrees of freedom used for very detailed finite element or computational fluid dynamics analysis. Reducing the computational cost reduces the time required for each time-domain simulation and allows for an increased number of experiments.

The structural equations are reduced using the normal mode method with the free-free vibration mode shapes. ${ }^{12}$ The displacement of the structure is represented as a linear combination of the mode shapes, i.e.,

$$
u(x, y, z, t)=\sum_{i=1}^{n} \Phi_{i}(x, y, z) \eta_{i}(t)
$$

where $u$ is the displacement vector, $\Phi_{i}$ is the mode shape of mode $i$, and $\eta_{i}$ is the amplitude of mode $i$. The free-free vibration mode shapes are eigenvectors and therefore form an orthogonal set of basis vectors to represent the displacement. The method reduces the structural equations to the number of degrees of freedom equal to the number of free-free vibration modes included $(n)$.

The aerodynamic loads are calculated using the vehicle outer mold line that is represented by a coarse mesh along the vehicle surface. The loads are calculated at each panel on the mesh using shock-expansion theory with the piston theory correction to account for unsteady flow, ${ }^{13,14}$

$$
\Delta p=\rho a^{2}\left(\frac{w}{a}+\frac{\gamma+1}{4}\left(\frac{w}{a}\right)^{2}+\frac{\gamma+1}{12}\left(\frac{w}{a}\right)^{3}\right)
$$

where $\Delta p$ is the increment in pressure due to unsteady flow, $\gamma$ the ratio of specific heats, $\rho$ the local density, $a$ speed of sound, and $w$ surface velocity perpendicular to the flow. This method eliminates the need to use CFD for the unsteady aerodynamics of the deforming and oscillating vehicle. However, piston theory 
assumes the uniform flow Mach number is sufficiently large and the deflection or the amplitude of surface oscillation is low.

The DACS system consists of multiple jets around the circumference of the vehicle nose that exhausts from the body surface perpendicular to the oncoming supersonic flow. Jets in supersonic crossflow have been studied experimentally and numerically and the results show that the jet introduces a very complex flow field around the vehicle. ${ }^{15,16}$ The effective force on the vehicle is a combination of the aerodynamic force without the jet, the force applied from the pressure and momentum transfer at the jet nozzle, as well as the jet interaction that causes regions of higher pressure fore and lower pressure aft of the nozzle. In the current study, only the first two effects are accounted for and the effective force from the DACS is modeled as additional pressure at the nozzle exit only. However, this approach neglects how the jet interaction flow structure might interact with the structural deformation.

The aeroelastic equations of motion are written as

$$
[M]\left\{\begin{array}{c}
\dot{\beta} \\
\dot{\zeta} \\
\ddot{\eta}
\end{array}\right\}+[C]\left\{\begin{array}{c}
\beta \\
\zeta \\
\dot{\eta}
\end{array}\right\}+[K]\{\eta\}=\left\{\begin{array}{c}
Q_{\beta} \\
Q_{\zeta} \\
Q_{\eta}
\end{array}\right\}
$$

for a flexible structure as presented by Frendreis and Cesnik. ${ }^{17}$ The vehicle dynamics are expressed in the body frame using the column vectors $\beta, \zeta$, and $\eta$. The quantity $\beta$ is a three-element column vector containing the $x, y$, and $z$ body frame translational velocities. The quantity $\zeta$ is a three-element column vector containing the body frame rotational velocities about the $x, y$, and $z$ axes. The column vector $\eta$ contains the amplitudes of each structural mode and its length corresponds to the number of modes chosen to represent the structure. At each time step in the simulation the external forces are calculated and used with the current state of the vehicle to integrate the equations of motion according to a fourth-order RungeKutta scheme.

\section{Numerical Modeling}

A new vehicle was created for this study and designed to be representative of slender high speed maneuverable vehicles. The basic dimensions and flight conditions for the vehicle are shown in Table 1 and are inspired by existing vehicles, such as the Sidewinder and AMRAAM. The baseline configuration in the study has a uniform mass and stiffness distribution, therefore the free-free vibration mode shapes are similar to a uniform free-free beam. The outer mold line of the model, shown in Figure 1, was chosen to move the center of pressure aft of the center of gravity based on the lack of fin surfaces that are conventionally located at the tail end of the vehicle. Markers for the center of pressure (C.P.) and center of gravity (C.G.) have been added to the figure based on the results of the baseline vehicle. Conventional fin surfaces are not included in this model due to their loss of effectiveness at high angles of attack. Instead, a DACS is located at $25 \%$ of the vehicle length. This system is assumed to be composed of reaction jets that provide additional forces normal to the body surface to control the vehicle orientation.

Table 1. Basic properties and flight conditions for the vehicle

\begin{tabular}{lrl}
\hline Property & Value & Unit \\
\hline Total mass (uniform distribution) & 85 & $\mathrm{~kg}$ \\
Length & 3 & $\mathrm{~m}$ \\
Diameter at mid-length & 0.13 & $\mathrm{~m}$ \\
Mach & $3-4$ & \\
Altitude & $40,000-60,000$ & $\mathrm{ft}$ \\
\hline
\end{tabular}

The structural configuration of the baseline vehicle was modified to study the impact of flexibility on vehicle response. As mentioned previously, the free-free vibration mode shapes were used to reduce the structural equations of motion. A finite element model was created to calculate the free-free vibration modes of the model given a mass and stiffness distribution. Only the first bending modes in the longitudinal plane under $200 \mathrm{~Hz}$ were used to reduce the system of equations. This set of modes should show the fundamental vehicle response to applied loads. 


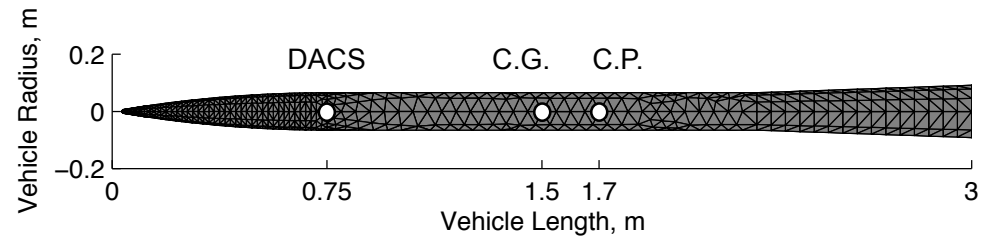

Figure 1. Side view of the undeformed axisymmetric vehicle

The first aspect of structural configuration that was analyzed is the overall vehicle stiffness. This was studied using a vehicle with a uniform mass distribution and free-free vibration mode shapes similar to the analytical solution of a uniform free-free beam. The stiffness of these mode shapes was then scaled to study the effect of flexibility on vehicle response. The range of free-free vibration frequencies used for the first bending mode is $25-40 \mathrm{~Hz}$. The stiff vehicle $(40 \mathrm{~Hz})$ is near the free-free vibration frequency of the Sidewinder vehicle and the flexible vehicle $(25 \mathrm{~Hz})$ represents the vehicle material stiffness value roughly cut in half. ${ }^{18}$

The second aspect of structural configuration was the distribution of stiffness along the length of the vehicle. The internal structure of the vehicle is nonuniform in general and this study investigates how different distributions of structural material or stiffness could lead to different responses. The internal structure of a high speed vehicle may have less support material surrounding the solid rocket fuel and more material surrounding the payload and control system. This variation in bending stiffness can been seen as either a change in thickness or material along the length of the vehicle. The bending stiffness distribution that was used to model the representative vehicle is shown in Figure 2. This distribution leads to a first bending mode shape with the inflection point aft of mid-length. A linear combination of the uniform and representative stiffness distribution shapes was used to derive several vehicle models. Each new set of vibration modes was mass normalized, the mass and inertia properties of the vehicle were kept constant at $85 \mathrm{~kg}$ uniformly distributed, and the lowest bending frequency associated with each was held constant to the lowest value of $25 \mathrm{~Hz}$. Figure 3 shows the free-free vibration mode shapes of the uniform distribution vehicle configuration 1, and Figure 4 shows the free-free vibration mode shapes of configuration 2. These two configurations were used as the bounds for generating new free-free vibration mode shapes.

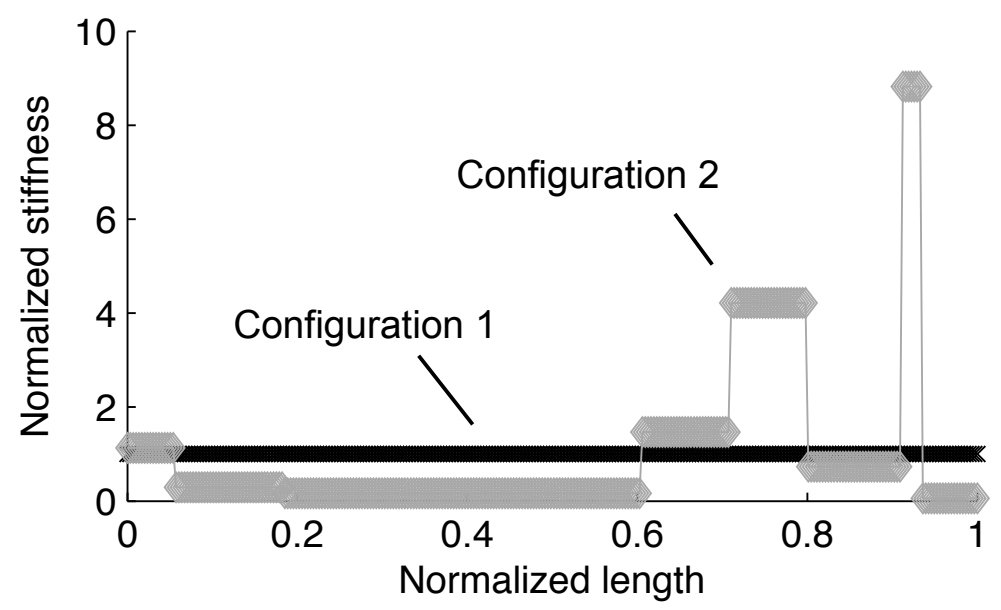

Figure 2. Normalized stiffness distributions of the uniform and representative vehicles 

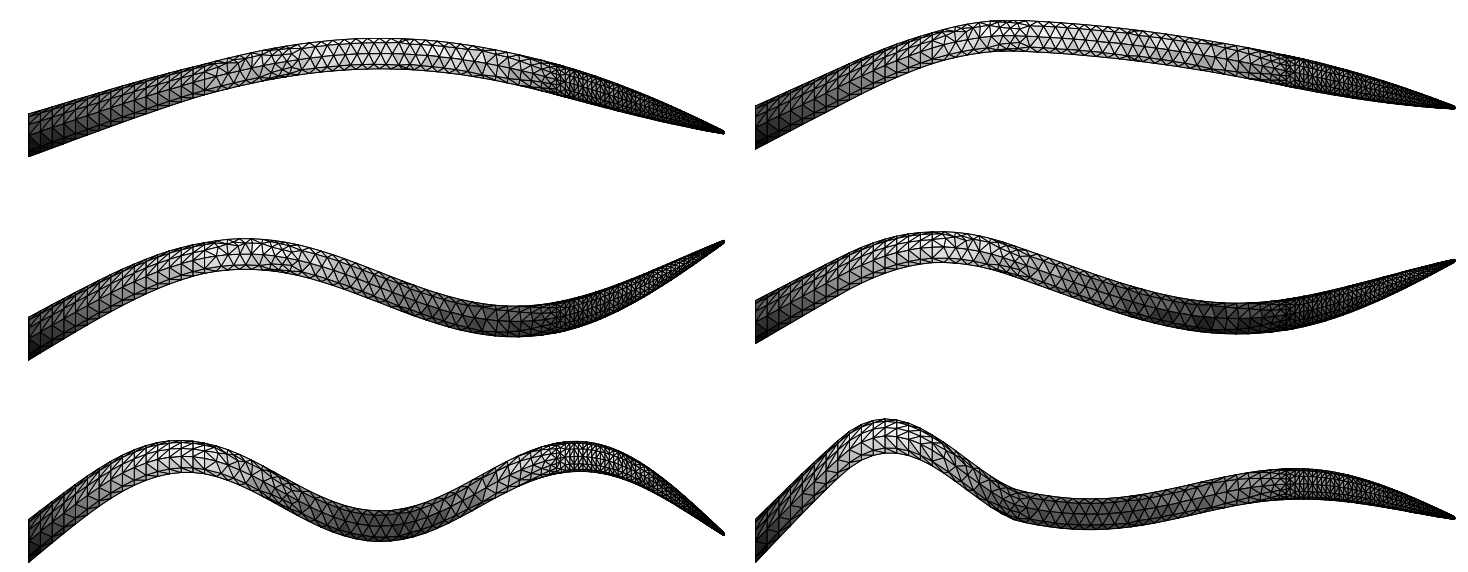

Figure 3. Free-free vibration mode shapes of the vehicle with uniform mass and stiffness (configuration 1)
Figure 4. Free-free vibration mode shape of the vehicle with uniform mass and representative stiffness distribution (configuration 2)

\section{Results}

Slender high speed vehicle response and performance during aggressive high speed maneuvers were measured using the reduced order vehicle and simulation framework. First, a steady aerodynamic analysis shows the variation of the aerodynamic loads for varying levels of vehicle deformation. Second, an open loop simulation with the DACS as the control input shows how the vehicle response is affected by varying the total stiffness from $40 \mathrm{~Hz}$ to $25 \mathrm{~Hz}$ and varying the stiffness distribution from configuration 1 to 2 . New mode shapes for the different stiffness distributions are calculated as

$$
\Phi_{i}=\Phi_{1 i}+\sigma\left(\Phi_{2 i}-\Phi_{1 i}\right) \quad \text { for } i=1 \ldots n
$$

where $\Phi_{i}$ is the $i$-th new shape, $\Phi_{1 i}$ is the $i$ - th configuration 1 shape, and $\Phi_{2 i}$ is the $i$ - th configuration 2 shape. The total number of mode shapes, $n$, used for the analysis is equal to 3 and corresponds to the first 3 free-free bending modes in the longitudinal plane. The configuration parameter $\sigma$ ranges from 0 to 1 to create new models that are linear combinations of configurations 1 and 2.

\section{A. Static aerodynamic results}

The aerodynamic characteristics of the model were evaluated about a set of steady conditions to measure the loads on the vehicle for the selected flight conditions. The loading on the vehicle was measured over a range of angles of attack from 0 to 60 degrees at Mach 3 and 40,000 ft for varying levels of deformation and different deformation shapes. All of the forces have been normalized by the free stream dynamic pressure, a reference length of $3 \mathrm{~m}$, and a reference area equal to $1 \mathrm{~m}^{2}$. The static margin was also calculated as the center of pressure changes for each aerodynamic flow condition and deformation. The static margin is calculated as:

$$
\text { Static Margin }=\frac{(\text { C.P. }- \text { C.G. })}{L_{r e f}}
$$

where $L_{r e f}$ is the reference length.

\section{Varying deformation}

The change in static aerodynamic properties of the vehicle with varying degrees of deformation is studied first. The results shown in Figure 5 were obtained using configuration 1 (Figure 3).

Figure 5 shows that vehicles with larger deformation have lower lift, higher drag, and higher moments about the center of gravity until about 30 to 35 degrees. This angle of attack is near the deflection angle for which an oblique shock will separate at Mach 3 in a two dimensional flow. Beyond 35 degrees the trend for drag flips so that the vehicles with larger deformation have lower drag. At zero angle of attack the vehicle 

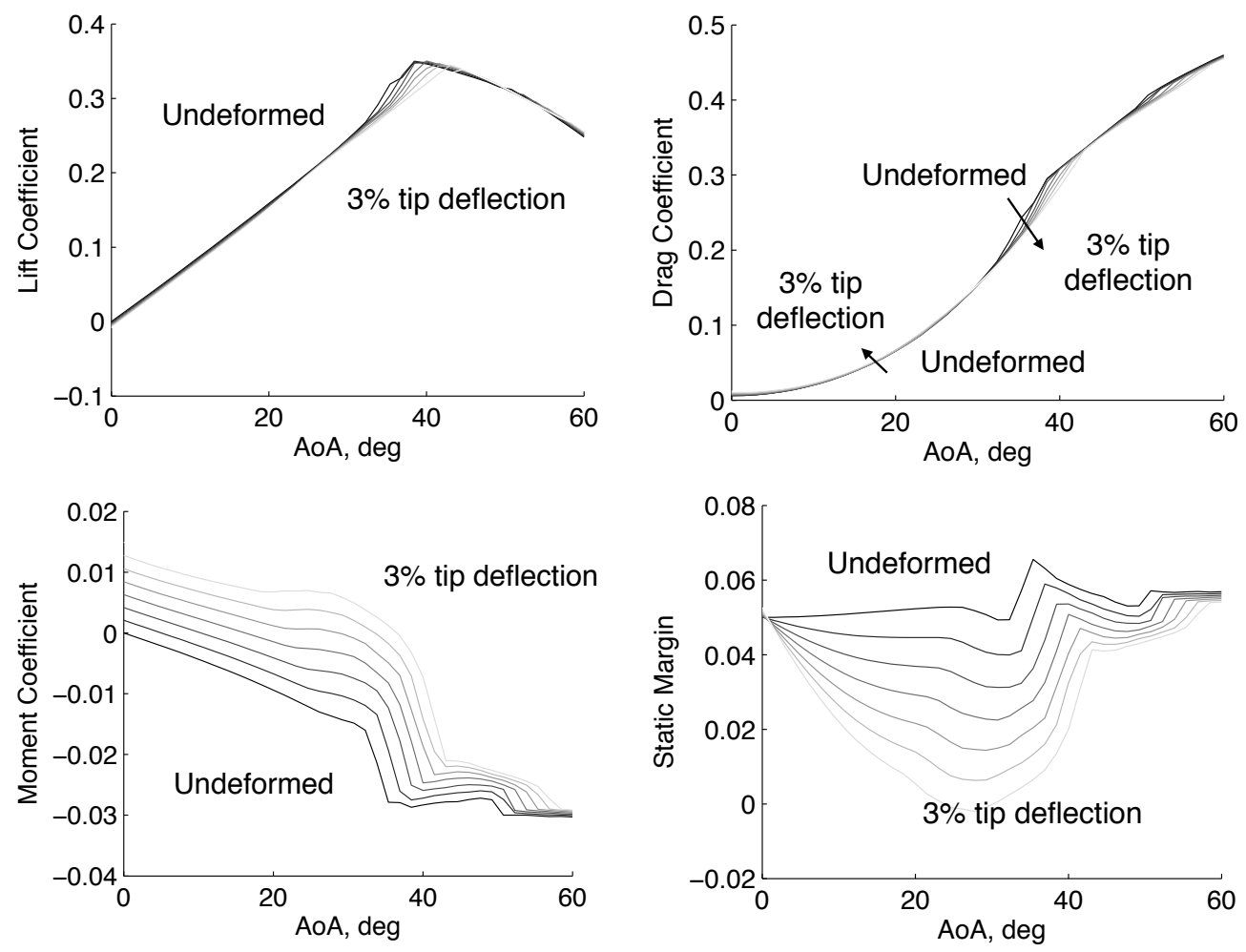

Figure 5. Flexible vehicle response with varying levels of deformation under static aerodynamic loading (configuration 1)

with higher deformation has a higher static margin. However, as the angle of attack and deformation are increased the static margin for the deformed vehicle is significantly decreased. This effect leads to deformed vehicles being more maneuverable at higher angles of attack.

\section{Varying stiffness distribution}

In reality the vehicle internal configuration can vary along the length and change the free-free vibration characteristics. The effects of the aerodynamic loading on the vehicle were studied as the stiffness distribution transitions to configuration 2. The effects on lift, drag and moment about the center of gravity are shown in Figure 6 for varying stiffness distribution. Only the first bending mode was included here and each mode shape is mass normalized thus a constant energy value was used as opposed to a constant tip deflection.

The results in Figure 6 show that configuration 2 has lower lift, higher drag, and higher moment at low angles of attack compared to configuration 1. At angles of attack larger than 40 degrees the trend for drag flips and configuration 2 has lower drag. The static margin plot shows the variation in mode shape for vehicles with $1 \%, 3 \%$, and $5 \%$ tip deflections. At lower angles of attack configuration 2 has a higher static margin. As the angle of attack is increased configuration 2 has a lower static margin than configuration 1 , which further increases the maneuverability of the vehicle.

\section{B. Vehicle response to DACS input}

The ability to quickly change the flight path to intercept a target is critical to success of the air-to-air vehicle and is measured in terms of maneuverability and agility. Maneuverability is defined here as the vehicle turn rate based on the flight path angle of the vehicle and the acceleration of the vehicle normal to the flight path. Agility has been measured using the time rate of change of turn rate. These parameters were chosen based on a previous study that compared the maneuverability and agility metrics across the industry. ${ }^{19}$ Two maneuvers were performed using the same DACS impulse over a short and long time duration. The

\section{7 of 16}

American Institute of Aeronautics and Astronautics

DISTRIBUTION A. Approved for public release, distribution unlimited. (96TW-2015-0344) 

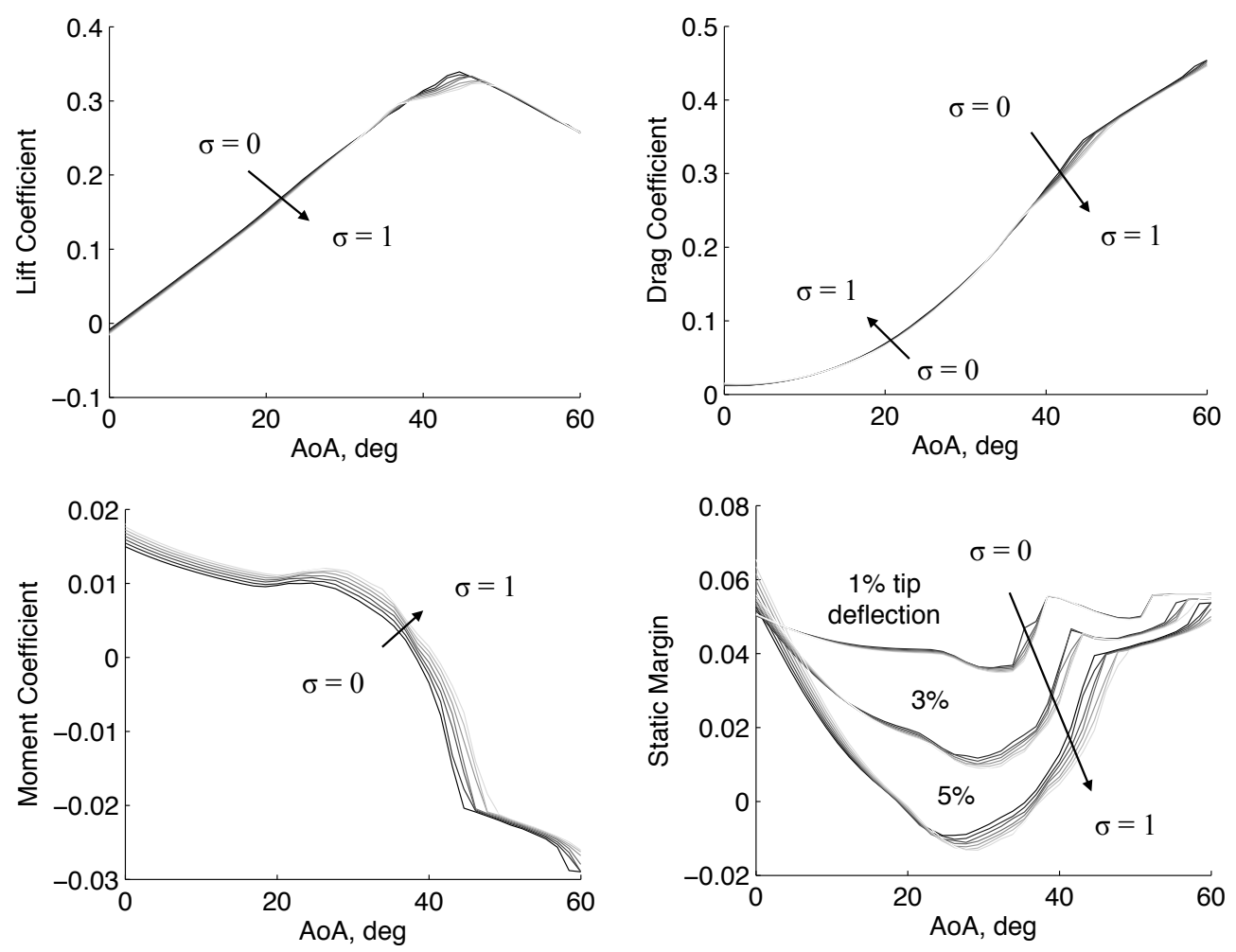

Figure 6. Flexible vehicle response under static aerodynamic loading with stiffness distribution varying from configuration 1 to 2

maneuvers used the DACS characteristics shown in Table 2 and applied according to

$$
F_{D A C S}= \begin{cases}\frac{I}{t_{d}} \sin (2 \pi f t) & 0<t<t_{d} \\ 0 & t>t_{d}\end{cases}
$$

Table 2. Properties of the DACS force applied to the vehicle

\begin{tabular}{|c|c|c|}
\hline Location & $25 \%$ length & \\
\hline Impulse, $I$ & $2500 \mathrm{~N} \cdot \mathrm{s}$ & \\
\hline Frequency, $f$ & $1 \mathrm{~Hz}$ & \\
\hline Application duration, $t_{d}$ & $0.5 \mathrm{~s}$ (short) & $1.5 \mathrm{~s}$ (long) \\
\hline
\end{tabular}

\section{Performance variation with stiffness}

High speed vehicles are often treated as rigid structures, but large aerodynamic loading implies the structural properties will have a significant impact on the simulation results. Choosing a range of stiffness values with the stiffness distribution of configuration 1 allowed us to investigate the impact of vehicle structural properties on the overall performance. A series of vehicles with varying flexibility have been modeled and the responses to the short jet impulse are shown in Figures 7 and 8. The stiff vehicle has an overall stiffness such that the first bending frequency is equal to $40 \mathrm{~Hz}$ while the flexible vehicle has a reduced stiffness such that the first bending frequency is $25 \mathrm{~Hz}$. Figures 9 and 10 show the vehicle response to the long duration impulse with varying levels of stiffness. This study is used to simulate a more complex maneuver to see if the trends for the short duration impulse still hold.

\section{8 of 16}

American Institute of Aeronautics and Astronautics

DISTRIBUTION A. Approved for public release, distribution unlimited. (96TW-2015-0344) 
The results show that flexibility has an effect on the vehicle response to a jet impulse used to turn the vehicle. The performance metrics follow trends shown in the steady aerodynamics results section. The vehicles with higher deformation have higher maneuverability and agility, but have lower velocities at the end of the maneuver. The improvements in the vehicle maneuverability and agility are summarized in Table 3 for the short and long duration impulses.

Table 3. Summary of performance percent increases by reducing the vehicle stiffness from $40 \mathrm{~Hz}$ to $25 \mathrm{~Hz}$

\begin{tabular}{rccc}
\hline Impulse duration & Normal acceleration & $\dot{\gamma}$ & $\ddot{\gamma}$ \\
\hline Short & $13.08 \%$ & $11.92 \%$ & $7.10 \%$ \\
Long & $23.09 \%$ & $21.48 \%$ & $13.13 \%$ \\
\hline
\end{tabular}
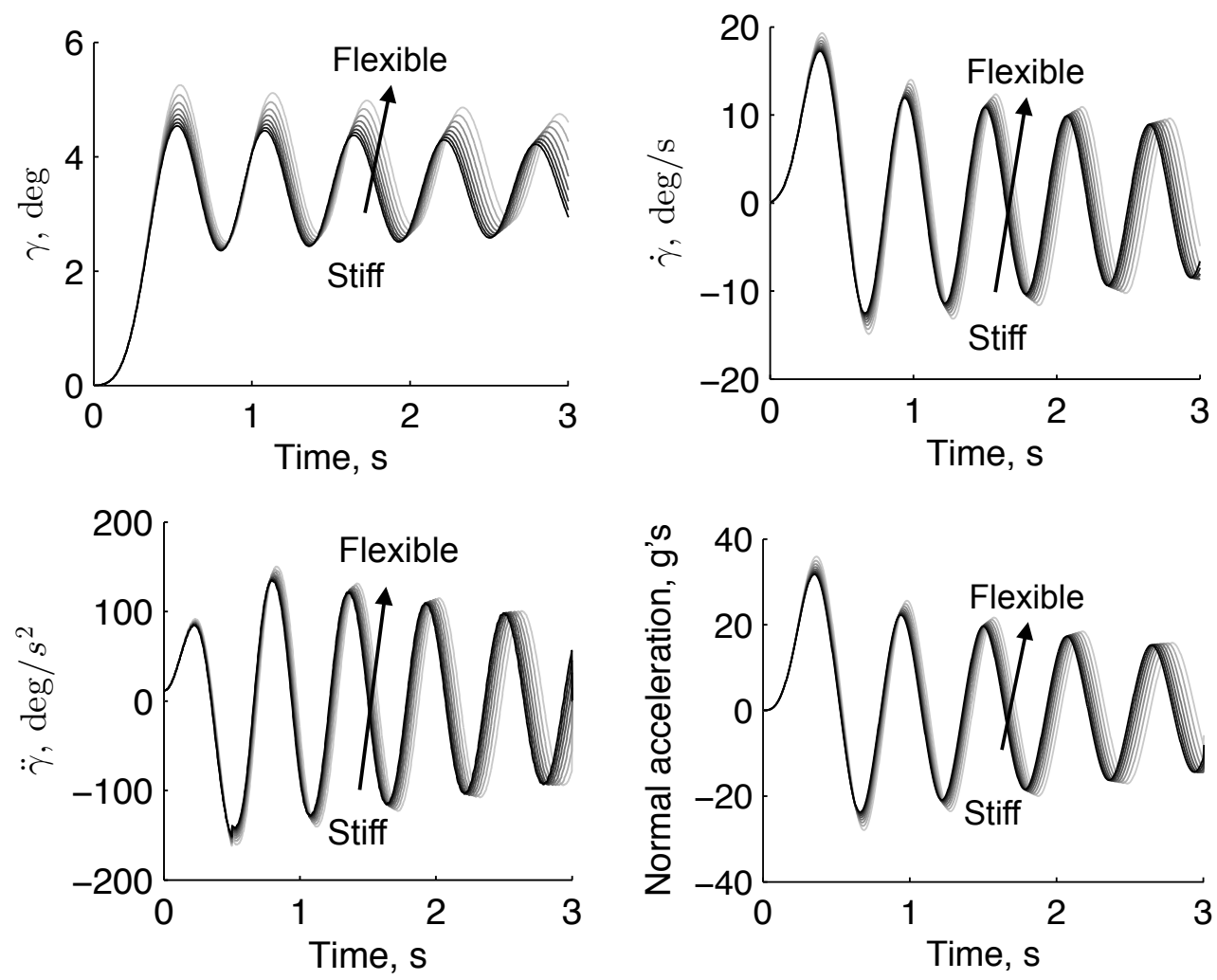

Figure 7. Performance results of the vehicle with varying stiffness in response to the short DACS impulse

\section{Performance variation with stiffness distribution}

The vehicle stiffness distribution was studied to measure its effect on vehicle performance. All of the vehicles have the same inertia properties and stiffness values, which isolates the difference in the results to the freefree vibration mode shapes. The lowest free-free vibration frequency $(25 \mathrm{~Hz})$ was used for all models as the mode shape is varied between configuration 1 and 2 shown in Figures 3 and 4, respectively. This shows the effects of moving the inflection point of the vehicle deformation and how this correlates to the previous work relating to rigid projectiles with a single degree of freedom. Figures 11-14 show the vehicle responses to short and long duration impulses with varying free-free vibration mode shapes.

The results show that the vehicles with the configuration 2 stiffness distribution are more maneuverable and agile than the vehicles with the uniform stiffness distribution. Similar to what was found in the steady aerodynamic results section the configuration 2 vehicles have higher drag and have lower velocities at the 

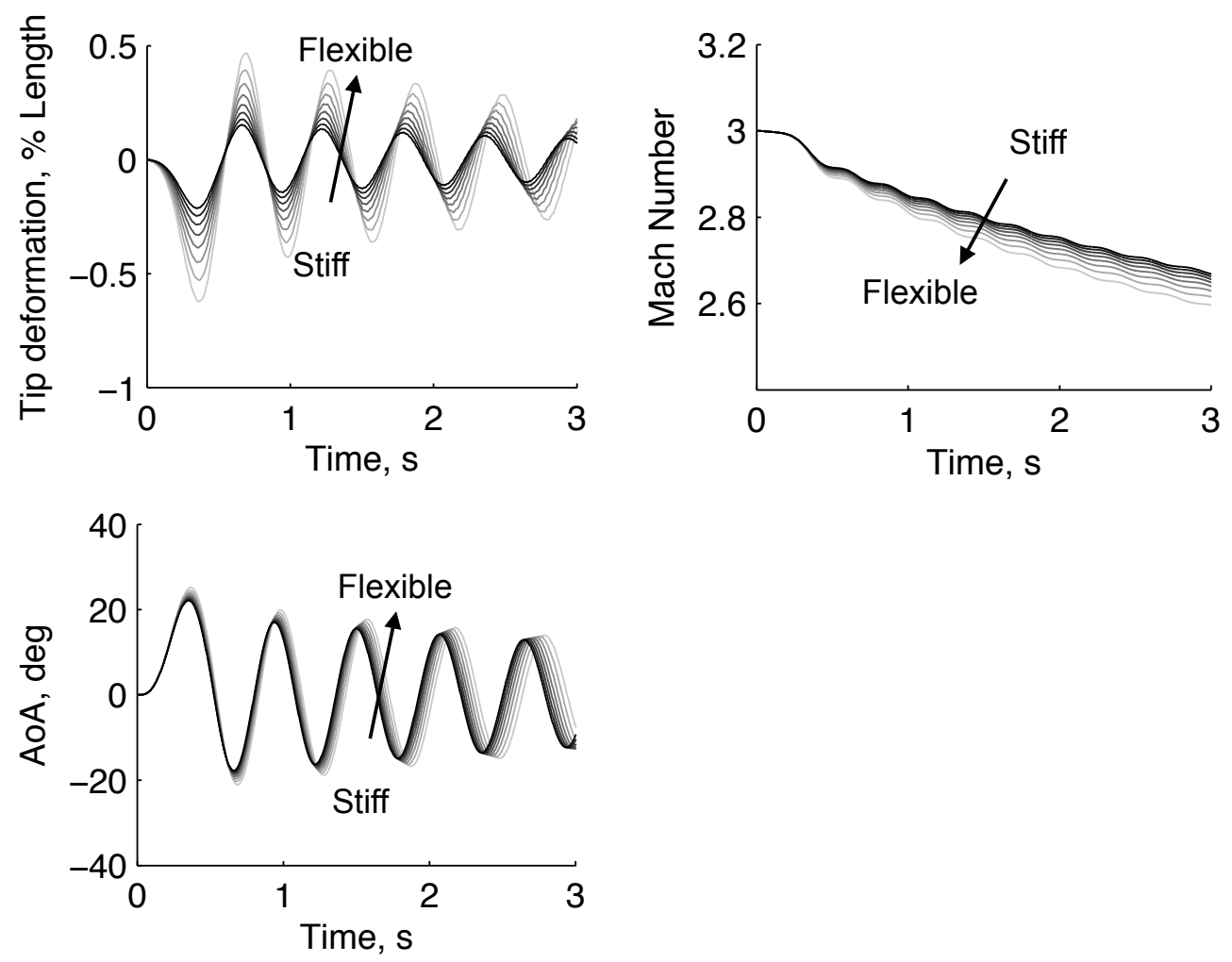

Figure 8. Structural and flight dynamics response of the vehicle with varying stiffness in response to the short DACS impulse

end of the maneuver. The improvements in the vehicle maneuverability and agility are summarized in Table 4 for the short and long duration impulses.

Table 4. Summary of performance percent increases by transitioning from configuration 1 to 2 for the $25 \mathrm{~Hz}$ case

\begin{tabular}{rccc}
\hline Impulse duration & Normal acceleration & $\dot{\gamma}$ & $\ddot{\gamma}$ \\
\hline Short & $2.58 \%$ & $2.34 \%$ & $2.45 \%$ \\
Long & $5.20 \%$ & $4.77 \%$ & $3.06 \%$ \\
\hline
\end{tabular}

\section{Discussion}

The focus of this study is the impact of flexibility, structural configuration, and reaction jet control inputs on the performance of slender high speed vehicles. Analyzing the results shows that even very small deformations ( $1 \%$ of the vehicle length) have a significant impact on the aerodynamic loading and vehicle response. In addition, the shape of the vehicle deformation has an impact on the vehicle loading and response. The results add to the findings in the literature by showing that the slender vehicle is more maneuverable as the inflection point of the free-free vibration mode shape is moved aft.

The static aerodynamic results provide the motivation to consider vehicle stiffness as a design parameter for improving vehicle maneuverability and agility. Considering the vehicle model with uniform properties, the vehicles with larger deformation have higher moment coefficients throughout the range of angles of attack that was considered. Therefore, a flexible vehicle will either reach higher angles of attack for the same applied force or will require less applied force to maintain the same angle of attack as compared to a stiffer vehicle. A similar effect is seen by considering the stiffness distribution of the vehicle. As the vehicle

\section{0 of 16}

American Institute of Aeronautics and Astronautics

DISTRIBUTION A. Approved for public release, distribution unlimited. (96TW-2015-0344) 

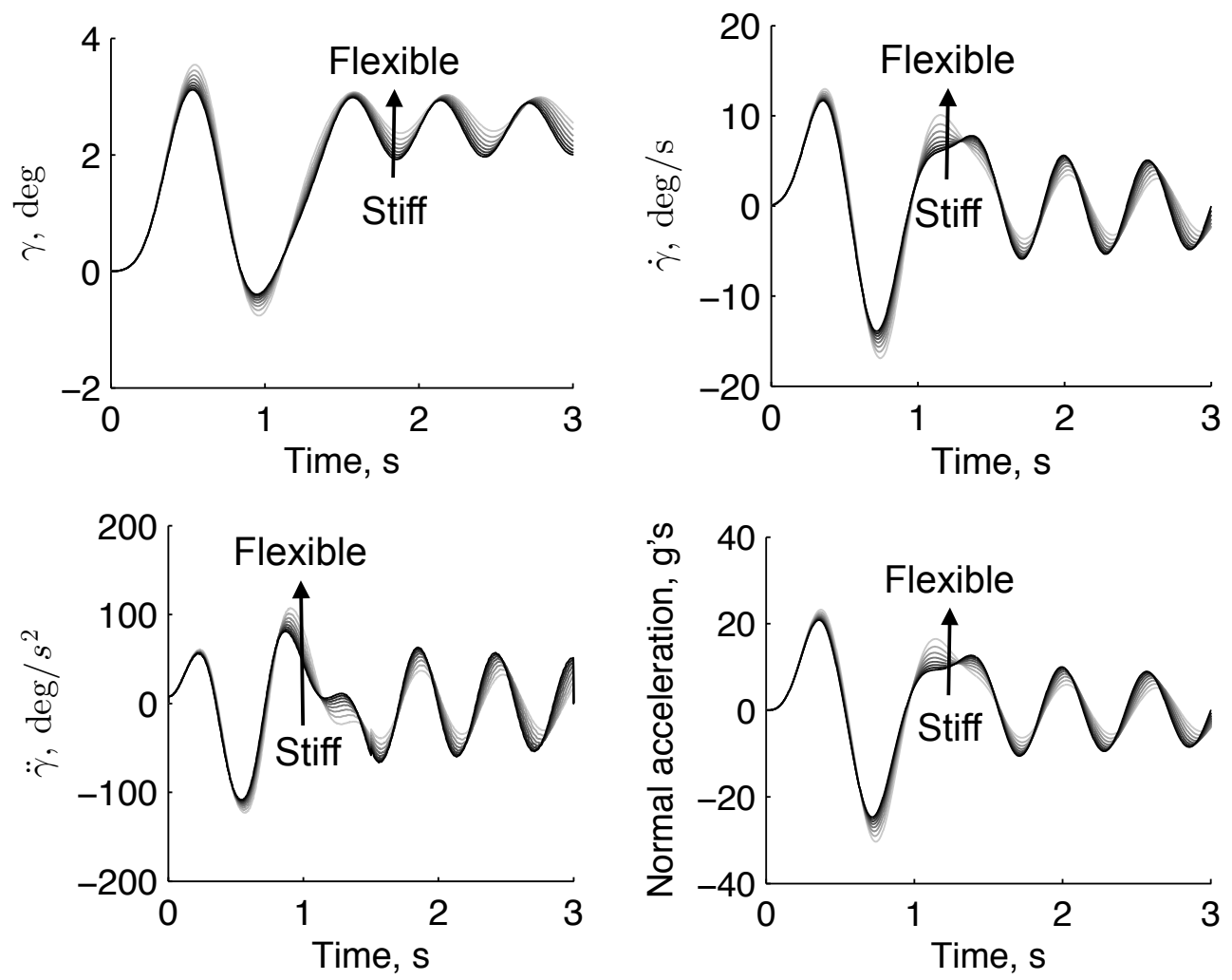

Figure 9. Performance results of the vehicle with varying stiffness in response to the long DACS impulse

shape is transitioned from a uniform stiffness distribution (configuration 1) to the representative stiffness distribution (configuration 2) the moment coefficient about the center of gravity is increased for the range of angles of attack that were measured. Therefore for the same amount of energy applied to the structure the vehicles with inflection points located aft will reach higher angles of attack. During the trajectory the vehicle is likely to have low deformation at low angles of attack and larger deformation at higher angles of attack. The static margin was decreased in both cases either by increasing deformation or moving the inflection point of the deformation aft. Therefore, the flexible vehicle for either configuration will have a little to no effect on stability during minor maneuvers, but will have a positive benefit on maneuverability during aggressive maneuvers by moving the center of pressure forward.

The vehicle response to the DACS impulse follows similar trends to the static aerodynamic results for vehicles with varying stiffness values and distributions. Considering the short and long duration impulses applied to the uniform vehicle with varying stiffness the flexible vehicles have larger deformations which translates to higher maneuverability and agility measured using the flight path angle. In addition, there is a phase shift that occurs in the vehicle response that stems from the flexible vehicles maintaining larger deformations for a longer time duration. Using the most flexible model then transitioning to the configuration 2 stiffness distribution further increases maneuverability and agility.

The results that have been presented show that vehicle maneuverability and agility are dependent on vehicle structural properties. The largest gains in performance are made as the structural configuration approaches a more flexible design. Additional gains are achieved by a structural configuration that moves the inflection point of the dominant deformation mode aft along the vehicle. In all these cases there is a common trade between maneuverability and vehicle velocity. This may be taken into consideration to design a vehicle with a mission to intercept a target. Regardless of application this work has shown that the coupling between the aerodynamics, structure and flight dynamics must be considered for this class of slender high speed vehicles. 

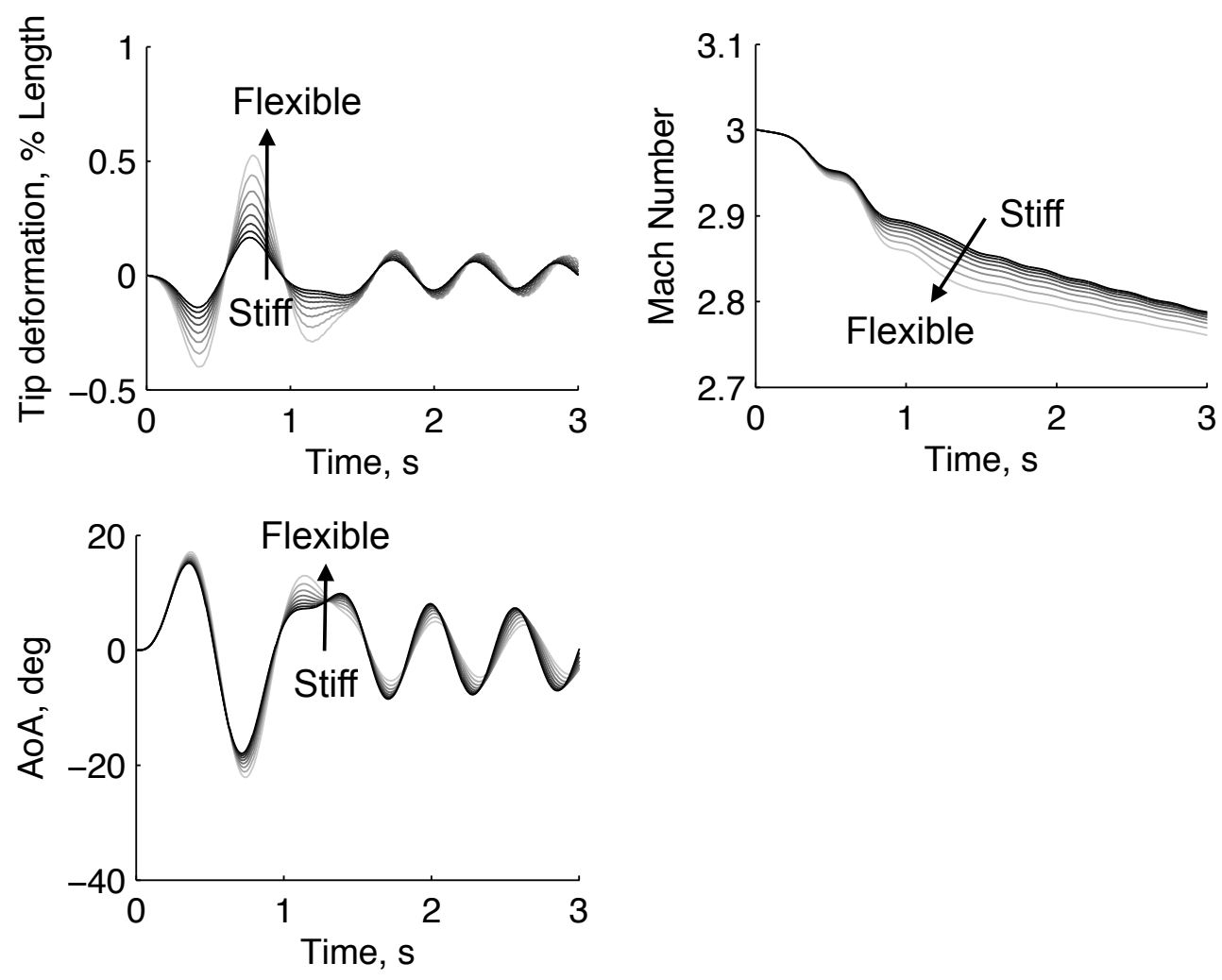

Figure 10. Structural and flight dynamics response of the vehicle with varying stiffness in response to the long DACS impulse

\section{Acknowledgments}

This research was sponsored by the Air Force Research Laboratory with Crystal Pasiliao and Daniel Reasor as the technical monitors. Opinions, interpretations, conclusions, and recommendations are those of the authors and are not necessarily endorsed by the U.S. Government.

\section{References}

${ }^{1}$ Wise, K. A. and Broy, D. J., "Agile Missile Dynamics and Control," AIAA Journal of Guidance, Control and Dynamics, Vol. 21, No. 3, May-June 1998, pp. 441-449.

${ }^{2}$ Costello, M. and Argarwalla, R., "Improved Dispersion of a Fin Stabilized Projectile Using a Passive Movable Nose," Atmospheric Flight Mechanics Conference, AIAA 2000-4197, 2000.

${ }^{3}$ Yao, S., Wu, Z., and Yang, C., "Trajectory Simulation of Flexible Missiles and the Effect of Flexibility on Hit Precision," AIAA Atmospheric Flight Mechanics Conference, AIAA 2014-0888, January 2014.

${ }^{4}$ Roger, R. P., "The Aerodynamics of Jet Thruster Control for Supersonic/Hypersonic Endo-Intercepters: Lessons Learned," 37th AIAA Aerospace Sciences Meeting and Exhibit, AIAA 99-0804, January 1999.

${ }^{5}$ Morton, S. A., McDaniel, D. R., Sears, D. R., Tillman, B., and Tuckey, T. R., "Kestrel - A Fixed Wing Virtual Aircraft Product of CREATE Program," 47th AIAA Aerospace Sciences MeetingIncluding The New Horizons Forum and Aerospace Exposition, AIAA 2009-338, January 2009.

${ }^{6}$ Skujins, T. and Cesnik, C. E. S., "Reduced-Order Modeling of Unsteady Aerodynamics Acorss Multiple Mach Regimes," AIAA Journal of Aircraft, Vol. 51, No. 6, November-December 2014, pp. 1681-1704.

${ }^{7}$ McNamara, J. J., Crowell, A. R., Friedmann, P. P., Glaz, B., and Gogulapati, A., "Approximate Modeling of Unsteady Aerodynamics for Hypersonic Aeroelasticity," AIAA Journal of Aircraft, Vol. 47, No. 6, November-December 2010, pp. 19321945.

${ }^{8}$ Crowell, A. R. and McNamara, J. J., "Model Reduction of Computational Aerothermodynamics for Hypersonic Aerothermoelasticity," AIAA Journal, Vol. 50, No. 1, January 2012, pp. 74-84.

${ }^{9}$ Baker, M. L., Alston, K. Y., and Munson, M. J., "Integrated Hypersonic Aeromechanics Tool (IHAT)," 9th AIAA/ISSMO Symposium on Multidisciplinary Analysis and Optimization, AIAA 2002-5512, September 2002.

\section{2 of 16}

American Institute of Aeronautics and Astronautics

DISTRIBUTION A. Approved for public release, distribution unlimited. (96TW-2015-0344) 

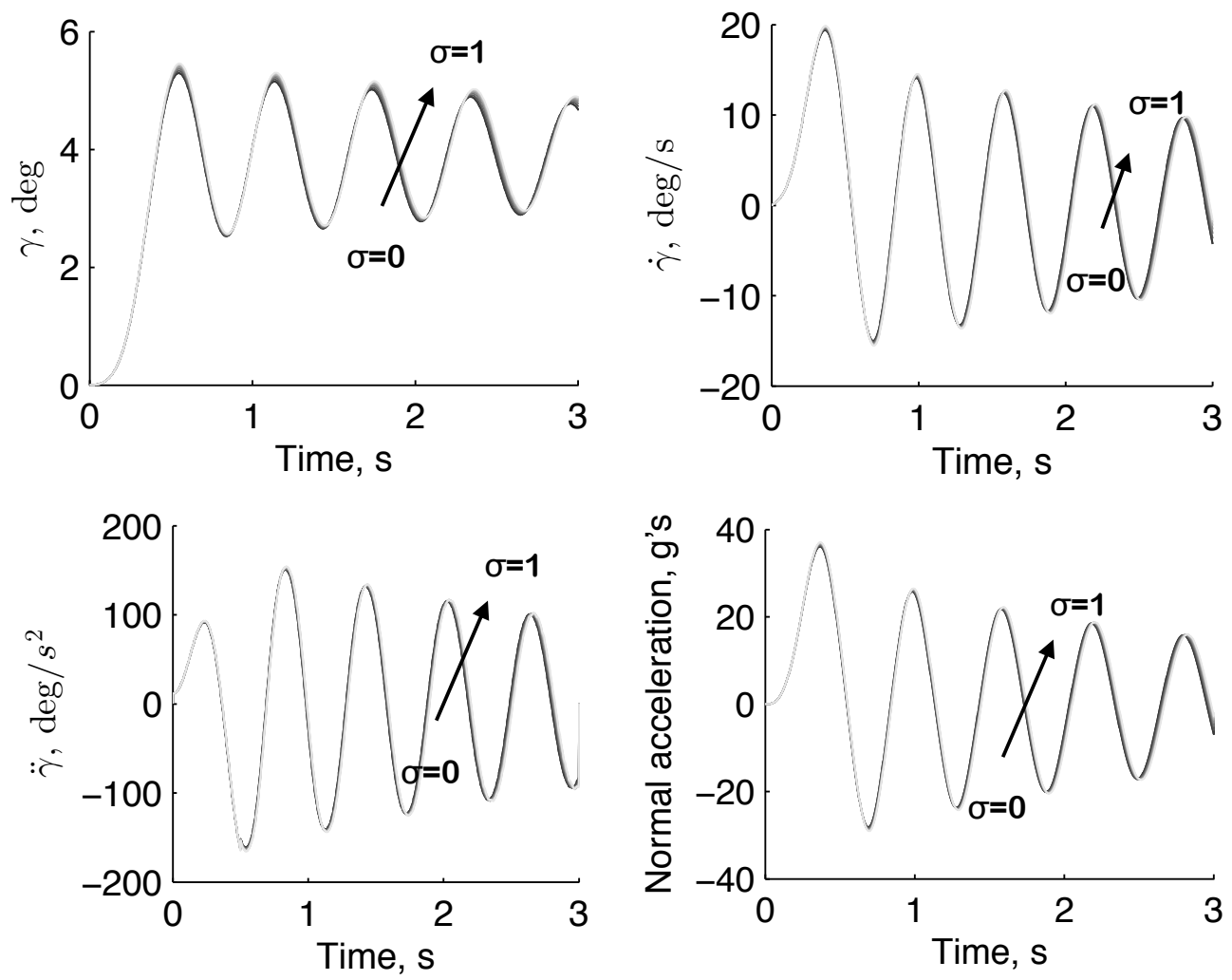

Figure 11. Performance results of the vehicle in response to the short DACS impulse with stiffness distribution varying from configuration 1 to 2 with the first bending frequency equal $25 \mathrm{~Hz}$

\footnotetext{
${ }^{10}$ Pasiliao, C. L., Sytsma, M. J., Neergaard, L. J., Witeof, Z. D., and Trolier, J. W., "Preliminary Aerothermal Structural Simulation," 14th AIAA Aviation Technology, Integration, and Operations Conference, AIAA 2014-2292, June 2014.

${ }^{11}$ Klock, R. J. and Cesnik, C. E. S., "Aerothermoelastic Simulation of Air-Breathing Hypersonic Vehicles," 55th AIAA/ASME/ASCE/AHS/SC Structures, Structural Dynamics, and Materials Conference, AIAA 2014-0149, January 2014.

${ }^{12}$ Craig Jr., R. R. and Kurdila, A. J., Fundamentals of Structural Dynamics, John Wiley \& Sons, Inc., 2006.

${ }^{13}$ Anderson Jr., J. D., Modern Compressible Flow With Historical Perspective, McGraw-Hill, Inc., 1990.

${ }^{14}$ Ashley, H. and Zartarian, G., "Piston Theory - A New Aerodynamic Tool for the Aeroelastician," Journal of the Aeronautical Sciences, Vol. 23, 1956, pp. 1109-1118.

${ }^{15}$ Spaid, F. W. and Zukoski, E. E., "A Study of the Interaction of Gaseous Jets from Transverse Slots with Supersonic External Flows," AIAA Journal, Vol. 6, No. 2, February 1968, pp. 205-212.

${ }^{16}$ Graham, M. J. and Weinacht, P., "Numerical Simulation of Lateral Control Jets," 37th AIAA Aerospace Sciences Meeting and Exhibit, AIAA 99-0510, January 1999.

${ }^{17}$ Frendreis, S. G. V. and Cesnik, C. E. S., "3D Simulation of Flexble Hypersonic Vehicles," AIAA Atmospheric Flight Mechanics Conference, AIAA 2010-8229, 2010.

${ }^{18}$ Shutty, M. A., Dynamic Modeling and Modal Analysis of an Air-to-Air Missile, Master's thesis, Naval Postgraduate School, September 1991.

${ }^{19}$ Bitten, R., "Qualitative and Quantitative Comparison of Government and Industry Agility Metrics," AIAA Journal of Aircraft, Vol. 27, No. 3, 1989, pp. 276-282.
}

13 of 16

American Institute of Aeronautics and Astronautics

DISTRIBUTION A. Approved for public release, distribution unlimited. (96TW-2015-0344) 

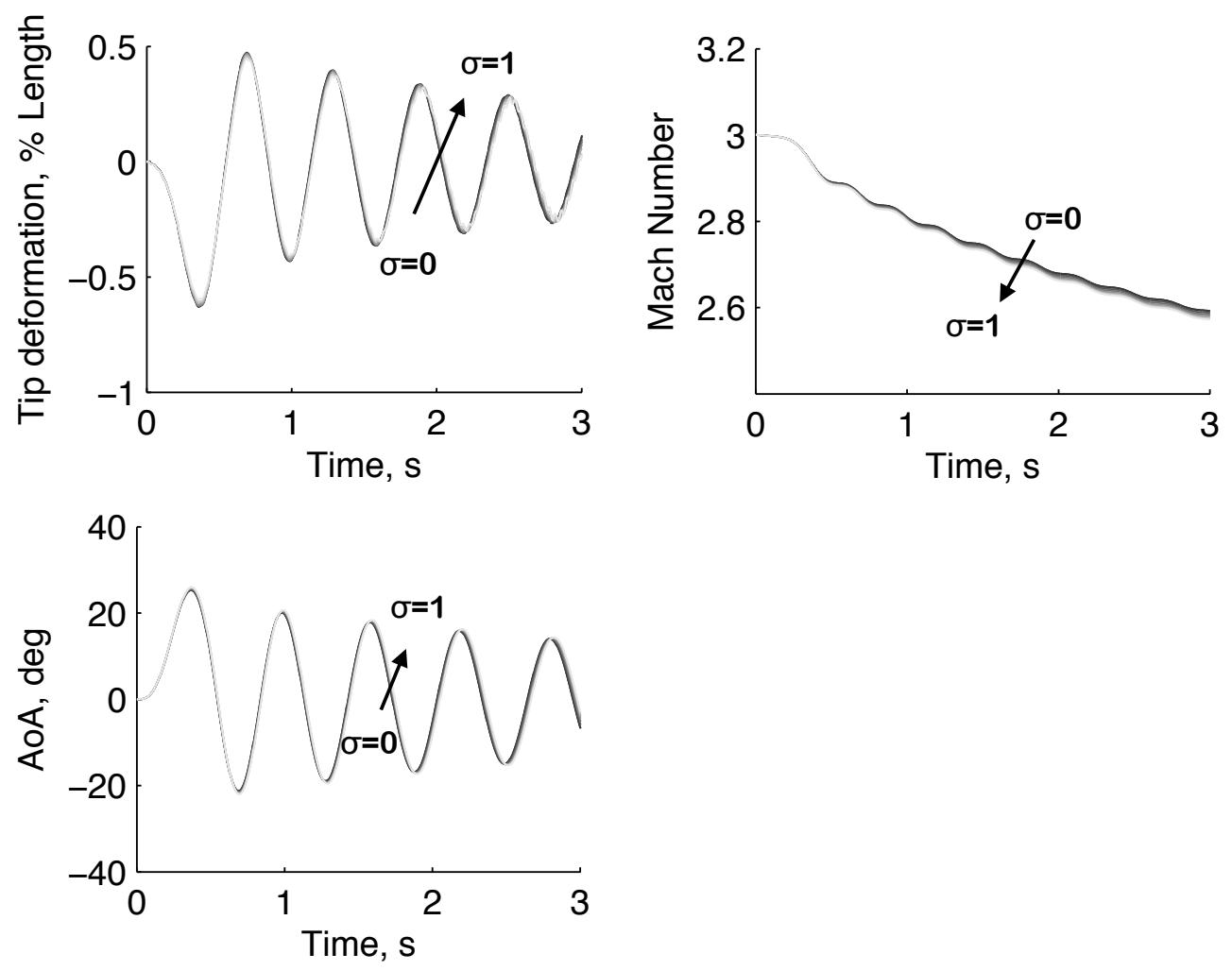

Figure 12. Structural and flight dynamics response to the short DACS impulse with stiffness distribution varying from configuration 1 to 2 with the first bending frequency equal $25 \mathrm{~Hz}$

\section{4 of 16}

American Institute of Aeronautics and Astronautics

DISTRIBUTION A. Approved for public release, distribution unlimited. (96TW-2015-0344) 

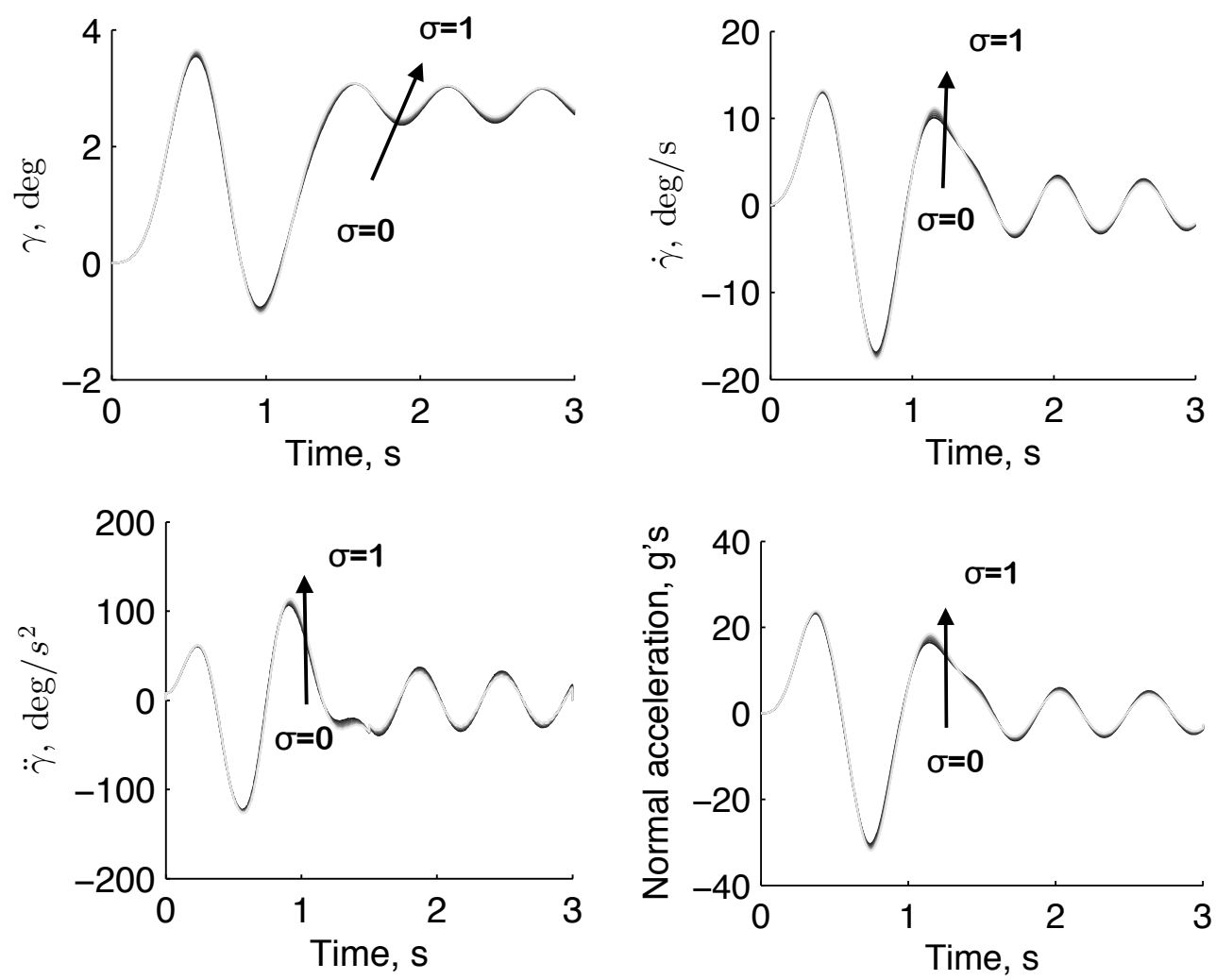

Figure 13. Performance results of the vehicle in response to the long DACS impulse with stiffness distribution varying from configuration 1 to 2 with the first bending frequency equal $25 \mathrm{~Hz}$ 

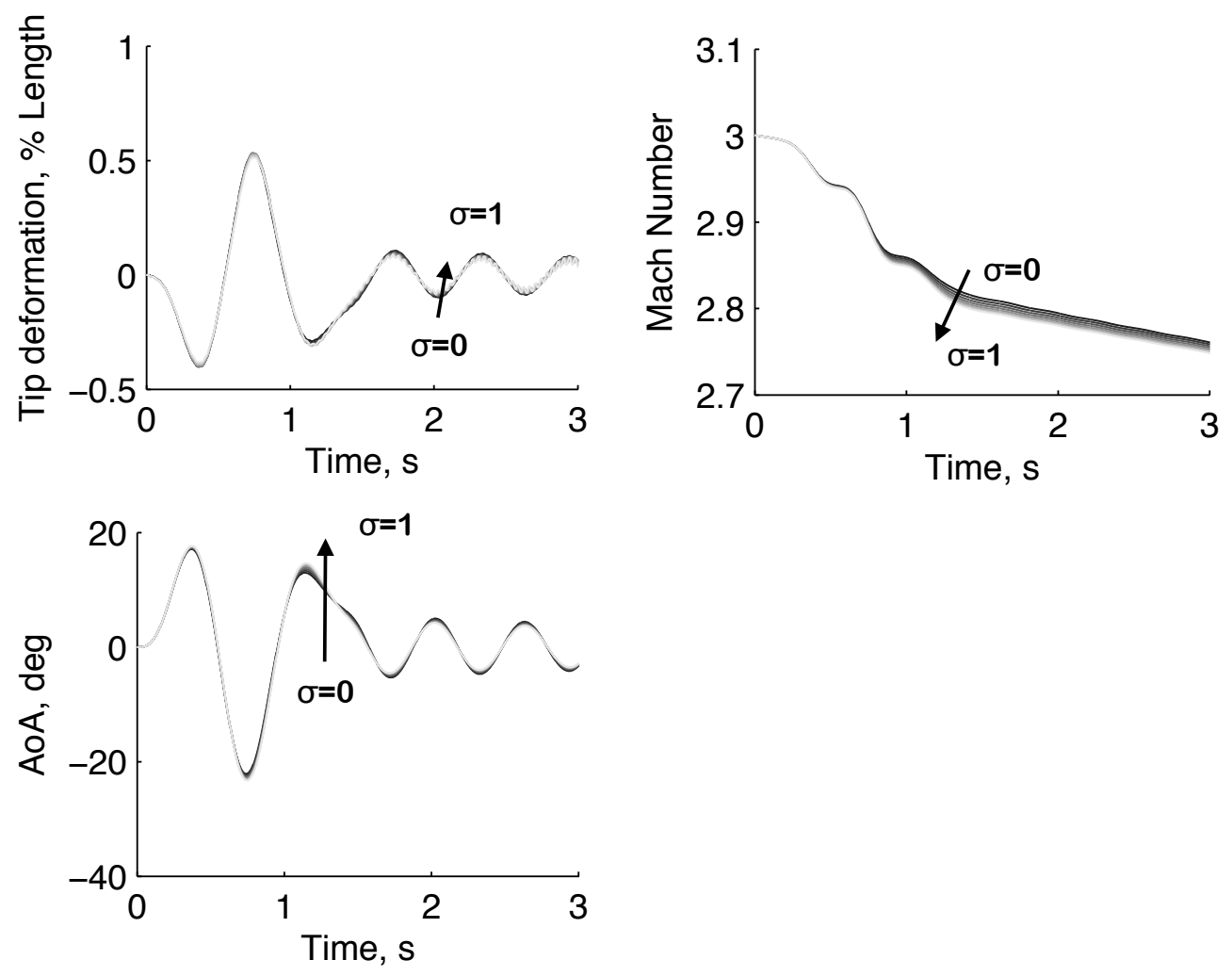

Figure 14. Structural and flight dynamics response to the long DACS impulse with stiffness distribution varying from configuration 1 to 2 with the first bending frequency equal $25 \mathrm{~Hz}$

\section{6 of 16}

American Institute of Aeronautics and Astronautics

DISTRIBUTION A. Approved for public release, distribution unlimited. (96TW-2015-0344) 